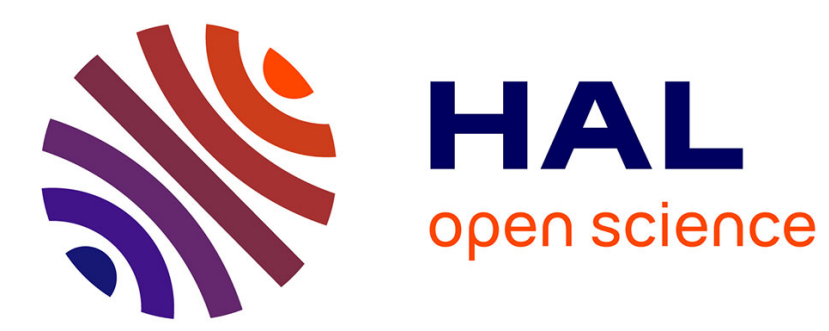

\title{
Restauration et invention : les célébrations du centenaire de la mort de Dante à Ravenne (1921)
}

Thomas Renard

\section{To cite this version:}

Thomas Renard. Restauration et invention: les célébrations du centenaire de la mort de Dante à Ravenne (1921). Histoire de l'art, 2011. hal-01858397

HAL Id: hal-01858397

https://hal-univ-rennes1.archives-ouvertes.fr/hal-01858397

Submitted on 7 Sep 2018

HAL is a multi-disciplinary open access archive for the deposit and dissemination of scientific research documents, whether they are published or not. The documents may come from teaching and research institutions in France or abroad, or from public or private research centers.
L'archive ouverte pluridisciplinaire HAL, est destinée au dépôt et à la diffusion de documents scientifiques de niveau recherche, publiés ou non, émanant des établissements d'enseignement et de recherche français ou étrangers, des laboratoires publics ou privés. 


\section{Thomas Renard}

\section{Restauration et invention : les célébrations du centenaire de la mort de Dante à Ravenne} (1921).

Dans la nuit du 13 au 14 septembre 1321, Dante Alighieri mourait en exil à Ravenne. Six cents ans plus tard, la ville qui avait accueilli le poète commémora en grande pompe cet anniversaire par la restauration d'un ensemble d'édifices. Cette célébration s'insérait dans le cadre d'un vaste programme promu par le gouvernement dès 1913 et qui conduisit à restaurer à partir de 1918 des monuments associés à Dante sur l'ensemble du territoire italien ${ }^{1}$. A Ravenne, le projet comprenait la reprise de divers édifices regroupés autour de la tombe de Dante (le quadrilatère de Braccioforte, l'église de San Francesco ainsi qu'un de ses cloîtres) définissant ce qui fut par la suite appelé la zona dantesca (fig. 1) ${ }^{2}$. En-dehors de cette zone, le programme de restauration embrassait un ensemble de bâtiments éparpillés à divers endroits de la ville et considérés comme ayant eu trait à la vie ou au temps de Dante : l'église de San Giovanni Evangelista, le Palazzo Veneziano del Comune (l'hôtel de ville de Ravenne) et la casa Traversari. Afin de «faire revivre la Ravenne de Dante ${ }^{3}$ », on restaura également les fresques du XIV ${ }^{\mathrm{e}}$ siècle des églises de San Giovanni Evangelista, de Santa Chiara, de Santa Maria in Porto Fuori et de San Francesco. A ces restaurations s'ajouta la création d'une nouvelle salle (la sala di Dante) au sein de la bibliothèque Classense et l'ouverture d'un musée dédié à l'art ravennate du moyen âge dans l'ancien couvent de San Vitale ${ }^{4}$. Face à l'ampleur de ces travaux, nous avons choisis de traiter ici plus particulièrement des deux principaux chantiers de 1921, ceux des églises de San Giovanni Evangelista et de San Francesco.

\footnotetext{
${ }^{1}$ La seule publication traitant de ces restaurations dans leur ensemble reste l'ouvrage officiel : Il secentenario della morte di Dante : 1321-1921 : celebrazioni e memorie monumentali, Milan, Bestetti e Tumminelli, 1928. Le livre, publié de façon anonyme, a été composé par Alfredo Lensi, Corrado Ricci et Santi Muratori.

${ }_{2}^{2}$ M. G. Benini, Luoghi danteschi, La Basilica di San Francesco e la Zona Dantesca a Ravenna, Ravenne, Longo, 2003 ; M. G. Benini, « Celebrazione, evocazione, invenzione nella zona dantesca a Ravenna », dans M. G. Muzzarelli (dir.), Neomedievalismi : Recuperi, evocazioni, invenzioni nelle città dell'Emilia-Romagna, Bologne, CLUEB, 2007, p. 201-226.

${ }^{3}$ Il secentenario [...], op. cit., p. 60 .

${ }^{4}$ Hormis la publication officielle déjà évoquée, on trouve des références à ces travaux dans A. Annoni, L'opera della Soprintendenza ai monumenti della Romagna per il VI ${ }^{\circ}$ centenario dantesco, Milan, Bestetti e Tumminelli, 1921 ; A. Annoni, Ravenna monumentale per il Centenario di Dante, Bergame, Ist. italiano d'arti grafiche, 1921, Bollettino d'arte del Ministero della pubblica istruzione, série 2, VII, 1922, p. 336-342 ainsi que dans la revue publiée par le Comitato Cattolico Dantesco de Ravenne, Il VI Centenario Dantesco, bollettino bimestriale, Ravenne, 1914-1921.
} 
Ces restaurations s'inscrivaient dans un courant qui avait vu se multiplier dans le nord et le centre de l'Italie des opérations de mise en valeur de l'aspect médiéval des villes ${ }^{5}$. Nous verrons comment grâce à un ensemble d'acteurs clés - depuis le poète Giosuè Carducci jusqu'à l'historien d'art Corrado Ricci et en passant par l'architecte Alfonso Rubbiani l'Emilie-Romagne, s'imposa entre la fin des années 1880 et les années 1920 comme une sorte de laboratoire de ce mouvement «néo-médiévaliste » à vocation identitaire ${ }^{6}$. En 1921, cette tendance était encore davantage légitimée par la référence à Dante. En effet, au cours du XIX siècle, s'était développé un véritable culte autour du poète, devenu peu à peu le symbole de l'unité italienne et des valeurs associées au Risorgimento ${ }^{7}$. Dans ce contexte, les restaurations conduites à l'occasion des commémorations de 1921 s'inscrivirent dans le cadre plus large de la construction de la dimension culturelle de la nation italienne.

\section{Restauration et promotion « néo-médiévale » des villes du centre et du nord de l'Italie}

Le débat portant sur la restauration avait passionné l'Italie de la seconde moitié du XIX siècle. Largement influencée par les écrits de Viollet-le-Duc, la pratique d'une restauration visant à rétablir l'unité stylistique s'était cristallisée autour de l'achèvement d'importants édifices religieux : la façade de Santa Maria del Fiore et de Santa Croce à Florence, celle de San Petronio à Bologne, de la cathédrale de Milan, ou encore celle de la cathédrale de Naples par l'architecte Enrico Alvino inaugurée en $1905^{\circ}$. Cette même année, prenaient fin les restaurations menées pendant près de vingt ans par Luca Beltrami au Castello Sforzesco de Milan ${ }^{9}$. Avec cet élève de l'architecte Camillo Boito, s'affirmait une nouvelle conception de la restauration, où l'importance du document historique prévalait sur l'unité stylistique. Ce système l'avait conduit à reconstruire une tour complètement disparue du Castello Sforzesco, la Torre del Filarete, sur la seule base de la documentation historique. A partir de 1903 Beltrami reconstituait le campanile de Venise, effondré l'année précédente, suivant le

\footnotetext{
${ }^{5}$ G. Zucconi, « Neo-medievalismo e città », Urbanistica, 91, juin 1989, p. 37-45.

${ }^{6}$ Voir les études récentes regroupées dans M. G. Muzzarelli (dir.), op. cit.

${ }^{7}$ A. Ciccarelli, «Dante and Italian Culture from the Risorgimento to World War I », Dante Studies, 119, 2001, p. 125-154. Pour une étude classique : C. Dionisotti, «Varia fortuna di Dante », dans Id., Geografia e politica della letteratura italiana, Turin, Einaudi, 1967, p. 255-303. La conception dominante en 1921 est présentée dans G. Mazzoni, «Dante nel inizio e nel vigore del Risorgimento », dans F. Angelitti (dir.), Dante e l'Italia, Rome, Fondazione Marco Besso, 1921, p. 347-380.

${ }^{8}$ Voir A. Restucci (dir.), Storia dell'architettura italiana, L'Ottocento, vol. I, Milan, Electa, 2005, ainsi que la bibliographie, p. 699-700.

${ }^{9}$ A. Bellini, «Luca Beltrami architetto restauratore », dans L. Baldrighi (dir), Luca Beltrami architetto, Milano tra Ottocento e Novecento, Milan, Electa, 1997, p. 92-143.
} 
précepte qui rapidement avait fait l'unanimité : «dov'era e com'era » («où il était, comme il était »).

A côté de ces deux conceptions (restauration stylistique et historique), Camillo Boito, à l'occasion du quatrième congrès des architectes et ingénieurs italiens de 1883, puis dans une publication de 1893, avait énoncé un ensemble de nouveaux préceptes de la restauration ${ }^{10}$. Il proposait une approche du monument plus respectueuse de l'ensemble de son histoire. Regardant avec méfiance le travail des restaurateurs de son temps, il posait comme principe de toujours favoriser l'intervention la moins invasive possible. Cette vision de la restauration qui se posait elle-même comme une troisième voie entre les positions de Ruskin et de Violletle-Duc, eut un retentissement considérable en Italie ${ }^{11}$.

En Emilie-Romagne, les enjeux de la restauration furent redéfinis dans le bouillonnement du milieu culturel de la Bologne fin-de-siècle. Autour du poète Carducci - Vate della Terza Italia - et de l'université ${ }^{12}$, la ville cherchait un nouvel élan après la période des Etats pontificaux. Aux yeux des intellectuels des cercles proches de Carducci, le renouveau de la ville passait par la promotion de l'image de la cité médiévale et la mise en avant des idéaux de liberté communale qui lui était rattachée. L'architecte et restaurateur Alfonso Rubbiani se chargea de traduire ce programme dans la pierre ${ }^{13}$. Le premier chantier d'envergure fut celui de l'église de San Francesco de Bologne. Les collaborations mises en place à cette occasion, allait conduire Rubbiani à créer en 1899 une association, le Comitato per Bologna Storica ed Artistica couplée avec un groupement d'artiste, l'Aemilia Ars, tous deux largement influencés par les conceptions de Ruskin et de Morris ${ }^{14}$. Pendant plusieurs décennies la ville délégua au comité la mise en œuvre de la plupart des chantiers de restauration qui devaient aboutir à la redéfinition de l'image gothique du centre actuel de Bologne. La transformation opérée au

\footnotetext{
${ }^{10}$ Atti del quarto Congresso degli Ingegneri e degli Architetti italiani radunato in Roma nel gennaio del 1883, Rome, Tipografia Fratelli Centenari, p. 122-127. Les propositions de Boito adoptées lors du congrès sont développées dans : C. Boito, Questioni pratiche di belle arti. Restauri, concorsi, legislazione, professione, insegnamento, Milan, Hoepli, 1893, partiellement repris dans C. Boito, Conserver ou restaurer les dilemmes du patrimoine, traduit de l'italien par Jean-Marc Mandosio, Besançon, Éd. de l'Imprimeur, 2000.

${ }^{11}$ Sur Camillo Boito, voir notamment F. Castellani, G. Zucconi (dir.), Camillo Boito: un'architettura per l'Italia unita, Venise, Marsilio, 2000, G. Zucconi, L'invenzione del passato. Camillo Boito e l'architettura neomedievale, 1855-1890, Venise, Marsilio, 1997 et en français la préface de Françoise Choay dans C. Boito, op. cit., 2000.

${ }_{12}$ En 1888, Carducci et Ricci organisèrent une grande célébration pour le $800^{\circ}$ anniversaire de l'université, W. Tega (dir.), Lo Studio e la Città. Bologna 1888-1988, Bologne, Nuova Alfa, 1987.

${ }^{13}$ Sur Alfonso Rubbiani (1848-1913), voir notamment L. Bertelli, O. Mazzei (éd.), Alfonso Rubbiani e la cultura del restauro nel suo tempo (1880-1915), acte de la journée d'étude, Bologne, 12-14 nov. 1981, Milan, Angeli, 1986 ; F. Solmi, M. Dezzi Bardeschi (éd.), Alfonso Rubbiani: $i$ veri e i falsi storici, catalogue d'exposition, Casalecchio di Reno, Grafis, 1981.

${ }^{14}$ C. Bernardini, D. Davanzo Poli, O. Ghetti Baldi (dir.), Aemilia Ars. Arts \& Crafts 1898-1903 a Bologna, Milan, A + G, 2001.
} 
travers d'une cinquantaine de restaurations fut telle que l'action de Rubbiani suscita dès 1910 de fortes critiques ${ }^{15}$. Les réticences soulevées par l'action de Rubbiani s'expliquent en partie par la lutte du Comitato contre ceux qui, sous prétexte de modernisation et d'assainissement de la ville, n'hésitaient pas à tailler de grandes percées (sventramenti) dans le tissu historique. Rubbiani connaissait les travaux de l'homme politique belge Charles Buls ${ }^{16}$ et peut-être avaitil lu également les écrits de l'architecte et théoricien Camillo Sitte ${ }^{17}$ : au-delà du problème de la restauration, c'est la question du rôle du patrimoine dans le développement urbain qui était ici en jeu, par l'opposition entre les « amateurs des mémoires artistiques », représentés ici par le Comitato, et les «tenants des exigences positives du développement moderne $»^{18}$.

Le travail de Rubbiani à Bologne servit assurément de référence à son ami Corrado Ricci ${ }^{19}$ lorsque celui-ci obtint en 1897 la tête de la «speciale Soprintendenza ai Monumenti della Romagna », première Soprintendenza moderne d'Italie ${ }^{20}$. A cette époque les édifices de Ravenne végétaient dans un état de conservation lamentable dont Hippolyte Taine avait dressé le sombre portrait en 1866 :

«On revient vers Ravenne, et le spectacle est encore plus triste. On n'imagine pas une ville plus abandonnée, plus misérablement provinciale, plus déchue. [...] On aperçoit des vieilles tours roussies et lézardées, des restes de construction anciennes appropriés à de nouveaux usages $[\ldots]^{21} »$.

Le rôle de Soprintendente permit à Ricci, d'œuvrer à la revalorisation de sa ville natale par la restauration des monuments religieux du $\mathrm{V}^{\mathrm{e}}$ et $\mathrm{VI}^{\mathrm{e}}$ siècle. Le chantier ouvert dès 1898 à l'église de San Vitale fut sans conteste le plus important de son passage à la Soprintendenza et

\footnotetext{
${ }^{15}$ G. Bacchelli, Giu le mani dai nostri monumenti antichi : note critiche sui progetti dei nuovi lavori al Palazzo del Podesta in Bologna, Bologne, Stab. Poligrafico Emiliano, 1910.

${ }^{16}$ Son essai sur l'esthétique des villes (Bruxelles, Bruylant-Christophe, 1893) avait été traduit en italien dès 1902.

17 Sur la réception de Sitte, notamment en Italie se reporter aux différentes contributions contenus dans G. Zucconi (éd.), Camillo Sitte e i suoi interpreti, acte du colloque, Venise, 1990, Milan, Angeli, 1992.

${ }^{18}$ G. Giovannoni, «Vecchie città ed edilizia nuova », Nuova antologia, 249, 1913, p. 449. Pour une analyse de cette opposition dans le développement urbain en Italie mise en perspective avec le conflit soulevé à la même époque par Benedetto Croce entre méthode expérimentale et méthode historique, voir G. Zucconi, La città contesa, Dagli ingegneri sanitari agli urbanisti (1885-1942), Milan, Jaca Book, 1989, p. 93-106.

${ }^{19}$ Corrado Ricci (1858-1934) avait connu Rubbiani lors de ses études à Bologne et fut membre honoraire du Comitato per Bologna Storica Artistica dès sa création. Voir F. Canali, «Alfonso Rubbiani e Corrado Ricci amicissimi », dans Varni A. (dir.), I confini perduti : le cinte murarie cittadine europee tra storia $e$ conservazione, Bologne, Compositori, 2005, p. 193-204. Sur la figure de Corrado Ricci, se référer à la bibliographie du catalogue : A. Emiliani, C. Spadoni (dir.), La cura del bello: musei, storie, paesaggi per Corrado Ricci, catalogue d'exposition, Museo d'arte della città di Ravenna, Milan, Electa, 2008.

${ }^{20}$ M. Dezzi Bardeschi, «Corrado Ricci e la nascita della Soprintendenza di Ravenna (1897) », dans N. Lombardini, P. Novara, S. Tramonti (dir.), Corrado Ricci : nuovi studi e documenti, Ravenne, Società di studi ravennati, 1999, p. 55-70.

${ }^{21}$ H. Taine, Voyage en Italie, Florence et Venise, Paris, Hachette, vol. II, 1866, p. 208-209.
} 
nous permet de comprendre sa conception de la restauration ${ }^{22}$. La méthode d'intervention de Ricci restent en partie héritée de la pratique du $\mathrm{XIX}^{\mathrm{e}}$ siècle : destruction d'ajouts postérieurs (chapelle du Sacrement), reconstruction par analogie (l'autel disparu, la marqueterie de marbre), simplification et régularisation des surfaces ou encore isolement de l'édifice. Toutefois d'autres pratiques plaident pour une vision plus moderne de la restauration. Ainsi Ricci ne suivit pas l'écrasante majorité qui avait voté la suppression des fresques du XVIII ${ }^{\mathrm{e}}$ siècle lors d'un référendum organisé parmi les personnalités du monde des arts ${ }^{23}$. On peut lire dans le même sens son attachement aux enquêtes historiques, aux fouilles systématiques, à la documentation photographique des différentes étapes du chantier puis, une fois les travaux achevés, à la publication des résultats.

L'influence de Ricci sur les travaux du centenaire dantesque fut déterminante, alors même qu'il n'avait plus d'affectation officielle à Ravenne et qu'il avait abandonné son poste de Directeur général des Antiquités et Beaux Arts au ministère de l'Instruction publique ${ }^{24}$. Il gardait une emprise particulièrement forte sur la Soprintendenza ai Monumenti, responsable pour 1921 de la maîtrise d'œuvre des chantiers. Il avait choisit pour lui succéder au poste de Soprintendente l'architecte et historien du Trentin Giuseppe Gerola (1877-1938), auquel il avait préféré en 1920, pour mener à bien les travaux du centenaire, Ambrogio Annoni (18821954), architecte milanais, ancien élève de Luca Beltrami et de Gaetano Moretti à l'académie de Brera. Au moment où Annoni arrivait à Ravenne au début 1920 les travaux de San Giovanni Evangelista et de San Francesco avaient déjà démarrés. Il les reprit en main et réussit à les mener à bien grâce à son énergie et malgré le manque de personnel et de financement. Il élabora à cette occasion la première ébauche de sa conception de la restauration architecturale autour de son refus de la schématisation abstraite et de sa théorie dite du «cas par cas $»^{25}$. Cette réflexion, par certains aspects proche de celle de Giovannoni, s'en éloignait par la liberté laissée à l'architecte de déterminer pour chaque chantier une

\footnotetext{
${ }^{22}$ P. Novara (éd.), Corrado Ricci e il San Vitale di Ravenna, Antologia di scritti, Ravenne, Tonini, 2008. Ricci a peu écrit sur sa pratique de la restauration architecturale, une étude a été récemment menée à ce propos : A. M. Bertelé, Corrado Ricci e il restauro architettonico, mémoire de laurea soutenu à l'université de Bologne, sous la direction de Maria Rosaria Valazzi, 2003.

${ }^{23}$ Parmi les personnalités de premier plan qui votèrent pour la destruction des fresques du XVIII ${ }^{\mathrm{e}}$ siècle, on retrouve entre autre Berenson, Geymuller, Boito, Ojetti, Venturi ou encore D'Annunzio. Beltrami fut un des rares à se prononcer contre.

${ }^{24}$ Cette implication apparait clairement dans sa correspondance conservée à la Biblioteca Classense de Ravenne (BCR), notamment dans BCR, fonds Ricci, Carteggio monumenti, 1917 à 1921, ainsi que les cinq volumes de BCR, fonds Ricci, Carteggio centenario dantesco, vol. 1-5.

${ }^{25}$ A. Annoni, L'opera [...], op. cit., p. 3-6. Pour une étude de sa conception de la restauration architecturale, se reporter à N. Pirazzoli, Teorie e storia del restauro, Ravenne, Essegi, 1994, p. 212-223 et à son ouvrage A. Annoni, Scienza ed arte del restauro architettonico. Idee ed esempi, Milan, Edizioni Artistiche Framar, 1946.
} 
méthode ad hoc, dictée par le caractère propre de l'édifice ${ }^{26}$. Plus tard au travers de sa conception de "l'avvaloramento » (valorisation) il défendit une restauration qui participe à l'amélioration du cadre urbain dans lequel elle s'insère ${ }^{27}$. Les écrits d'Annoni, bien que peu connus en France, restent pour l'Italie, à côté de ceux de Gustavo Giovannoni, les plus importants pour la pensée de la restauration architecturale du début du XIX ${ }^{\mathrm{e}}$ siècle.

\section{San Giovanni Evangelista libérée de « l'injure des siècles »}

La tradition voulait que l'église de San Giovanni Evangelista, la plus ancienne de Ravenne, ait été fondée en 424 suite à un vœu de Galla Placidia formulé durant une tempête en mer. Dès 1913, Ricci avait souhaité intégrer la restauration de cet édifice dans le programme des commémorations du centenaire ${ }^{28}$. Gerola rédigea en 1917 un premier projet mais il fallut attendre 1919 pour que les travaux débutent ${ }^{29}$. Dans son rapport, Gerola soulignait l'impossibilité de ramener l'édifice à son état original par manque d'informations historiques mais aussi parce que certains ajouts postérieurs méritaient d'être conservés. Toutefois, au-delà de ce compromis, les travaux recherchèrent une harmonie d'ensemble, celle d'une architecture régulière, sévère et austère ${ }^{30}$. Une architecture principalement marquée par de grandes surfaces de briques nues scandées par quelques lésènes encadrant des ouvertures en plein cintre.

On élimina dans un premier temps l'ensemble des décorations de stuc du XVIII ${ }^{\mathrm{e}}$ siècle, alors décrite comme une injure des siècles et cachant la véritable forme de l'édifice (fig. 2$)^{31}$. Toutes les parties de l'édifice pouvant nuire à l'harmonie d'ensemble et jugées comme ne présentant pas un intérêt historico-artistique notable furent détruites ${ }^{32}$. Ainsi on démolit toutes les chapelles émergeant du flanc nord de l'édifice, à l'exception de celle dite de Giotto, en raison de son caractère historique la rattachant à Dante (elle avait était érigée à l'époque de

\footnotetext{
${ }^{26}$ Giovannoni durant les mêmes années établissait un ensemble de catégories d'intervention sur les monuments, différenciant les restaurations de consolidamento, ricomposizione, liberazione, completamento, et innovazione. G. Giovanni, Il restauro dei monumenti, Rome, Cremonese, 1945.

${ }^{27}$ A. Annoni, op. cit., 1946, p. 24.

${ }^{28}$ G. Mesini., Memorie del Centenario Dantesco (1921) e di altre opere dantesche, Ravenne, Arti Grafiche, 1959, p. 7.

${ }^{29}$ Le projet daté du 2 mai 1917 se retrouve dans Archivio Centrale dello Stato (ACS), AABBAA div.1 1908-24 1420 et Soprintendenza ai Monumenti della Romagna (SMR), Archivio Documenti, RA 41-307.

${ }^{30}$ Bollettino d'arte, art. cit., p. 336. Ces qualificatifs apparaissent de façon récurrente dans toutes les publications et documents d'archives liés au centenaire.

${ }^{31}$ G. Gerola, Projet du 2 mai 1917, cité note 29.

${ }^{32}$ A. Annoni, L'opera [...], op. cit., p. 9.
} 
l'exil du poète entre 1317 et 1321) et des traces de fresques du début du XIV siècle qui y avait été découvertes (fig. 4$)^{33}$.

Sur la façade on dégagea le prothyron entourant le portail central au-dessus duquel apparut une large bifore du $\mathrm{XVI}^{\mathrm{e}}$ siècle. Annoni décida de supprimer cette bifore considérant que sa taille excessive nuisait à l'harmonie d'ensemble de la façade ${ }^{34}$. A la place, une petite fenêtre fut construite sur le modèle de celles que l'on retrouve sur les côtés de la façade. L'ensemble des structures de l'édifice furent reprises et consolidées par l'introduction de béton dans les fondations. Avec la destruction des décors de stuc recouvrant les murs, on supprima également les voutes de la nef et des collatéraux afin de rendre la charpente visible. Les lunettes de la voute en berceau à croisée furent comblées et on rétablit une rangée régulière de fenêtres-hautes en plein-cintre reconstruites sur le modèle de celles de la basilique de SaintApollinaire in Classe. Des fouilles furent entreprises simultanément aux premières destructions. Celles-ci permirent de reprendre totalement le mur original de l'abside selon un plan à pans coupés (fig. 3). Dans ce mur, Annoni rétablit la galerie d'ouverture originale et abaissa la couverture de l'abside suivant les traces découvertes en cours de chantier. Les résultats des fouilles lui permirent également de reconstruire à sa hauteur d'origine l'arctriomphal en ciment-armé recouvert de briques. Enfin, un effort considérable fut déployé afin d'isoler le bâtiment et de l'offrir à la vue du passant, celui-ci se trouvant le long de l'axe reliant la gare à la place centrale de la ville ${ }^{35}$.

Malgré l'activité frénétique d'Annoni, les travaux ne furent pas achevés pour la célébration du centenaire en septembre 1921. Une cérémonie eut lieu dans l'église, mais celle-ci resta pendant plusieurs années sans portes ni fenêtres, ni même couverture au-dessus de l'abside. L'absence totale de fonds disponibles à la suite de l'immense programme de 1921 bloqua la reprise des travaux qui ne furent achevés qu'en 1925.

En-dehors de San Giovanni Evangelista, on restaura également les façades du Palazzo Veneziano del Comune (fig. 5), et de la Casa dei Traversari, privilégiant l'aspect sévère d'un mur de brique rehaussé de très rares touches de travertin et scandé de bifores intégrées dans des arcs en plein cintre ${ }^{36}$.

\footnotetext{
${ }^{33}$ Ibid. p. 9-15.

${ }^{34}$ BCR, fonds Ricci, Carteggio Monumenti, 1920, 86, lettre d'Annoni à Ricci,19 juillet 1920.

${ }^{35}$ Voir SMR, Archivio Documenti, RA 41-307.

${ }^{36}$ A. Annoni, Ravenna [...], op. cit., 1921, p. 15-17.
} 


\section{San Francesco : l'église des funérailles de Dante et la zona dantesca}

L'autre grand chantier de Ravenne pour la célébration du centenaire, la restauration de l'église de San Francesco, fut à l'origine même de l'idée de commémorer Dante par le monument ancien. L'initiative revenait au prêtre Giovanni Mesini qui dès 1912 avait souhaité faire revivre l'église des funérailles de Dante ${ }^{37}$. Cette restauration de l'église fut accompagnée du réaménagement de l'aire adjacente située au nord du bâtiment afin de créer une zone dédiée à la mémoire du poète.

L'église, selon le chroniqueur Andrea Agnello, avait été érigée sous la direction de l'évêque Néon entre 449 et $475^{38}$. Initialement dédiée aux apôtres, la Basilica Apostolorum devint propriété des frères mineurs en 1261. Elle connut plusieurs importantes campagnes de remaniement, jusqu'à être presque complètement reconstruite au cours des XVII ${ }^{\mathrm{e}}$ et XVIII ${ }^{\mathrm{e}}$ siècles. Ce dernier chantier, mené en 1793 par l'architecte Guglielmo Zumaglini élève de Camillo Morigia $^{39}$, modifia profondément l'ensemble de la décoration et de la structure de l'église ${ }^{40}$. Les photographies précédant le début des travaux en 1918 nous montrent une nef baignée de lumière grâce aux grandes lunettes du vaisseau central et aux élégantes décorations de stucs clairs aux motifs de festons et de médaillons (fig. 6). Ces images apparaissent fort différentes de la description que nous en fait l'historien d'art et archéologue Giuseppe Bovini en 1971: «Malgré l'extrême simplicité de ses lignes architecturales, l'intérieur de l'église, bien que nu et dépouillé, produit une profonde impression par la spiritualité mystique qui s'en dégage $^{41} »($ fig. 7$)$.

Cette métamorphose fut le résulta de l'action volontaire des restaurateurs de 1921. Pour ces derniers, San Francesco devait redevenir l'église des funérailles de Dante. Il s'agissait pour eux de faire revivre l'atmosphère des obsèques du poète, à laquelle ne pouvait convenir encore une fois que l'image d'une architecture simple, sévère et austère. Cette transformation n'était toutefois pas présentée comme une reconstruction, mais bien plus comme une remise à jour de la véritable église, débarrassée de son «triste voile baroque ${ }^{42}$. Dans l'expectative et

\footnotetext{
${ }^{37}$ Don Mesini (1879-1969) raconte lui-même, la préparation du centenaire dans G. Mesini, op. cit., p. 3-11.

${ }^{38}$ Voir G. Bovini, La «Basilica Apostolorum » attuale chiesa di S. Francesco di Ravenna, Ravenne, Longo, 1964, p. 6.

${ }^{39}$ Sur ce dernier, sans doute l'architecte le plus célèbre de Ravenne, voir Dezzi Bardeschi M, Fabbri P., Pirazzoli N., Camillo Morigia (1743-1795), architetture e riformismo nelle legazioni, Ravenne, Santerno, 1976.

${ }^{40}$ M. G. Benini, op. cit., 2003, p. 25.

${ }^{41}$ G. Bovini, Ravenne, Paris, édition du Cercle d'Art, 1971, p. 42. L'église fut partiellement détruite durant la Seconde Guerre mondiale mais reconstruite telle qu'elle pouvait apparaitre après 1921.

${ }^{42}$ Bollettino d'arte [...], op. cit., p. 337.
} 
l'excitation du chantier, la restauration prenait la forme d'une fouille archéologique où, sous les couches successives, devait émerger Dante et son église.

« On cherchait Dante; on voulait 'l'église de Dante'; ombre invisible et propitiatoire, Dante guidait les recherches, alimentait les espérances, suscitait les enthousiasmes. Il y avait dans le cours des travaux, comme l'attente d'un miracle ${ }^{43}$. »

De façon comparable à San Giovanni Evangelista, les travaux débutèrent en 1918 par un vaste chantier de fouille mené de front avec la suppression de toutes les parties considérées comme baroques. On élimina les enduits du clocher et de la façade afin de rendre visible le parement de brique. Le portail du XVII ${ }^{\mathrm{e}}$ siècle fut retiré, laissant une simple ouverture en plein-cintre, au-dessus de laquelle on perça une bifore. Le dernier étage du campanile, ajout tardif, fut démoli et remplacé par une grande ouverture composée de quatre baies jumelées surmontées de trois patères en majolique selon un parti complètement arbitraire ${ }^{44}$. Les enduits et les décors de stuc des parois intérieures furent également retirés, tout comme les voutes en berceau à croisée afin de rendre la charpente visible. Le plafond du vaisseau central fut abaissé et orné de caissons en lambris qui reçurent une décoration picturale sobre, de tonalité chaude et de saveur moyenâgeuse. La suppression des lunettes entraîna la reprise des fenêtres hautes entourées à l'extérieur de lésènes et de frises d'arceaux, soulignant encore la régularité de l'édifice.

Le chœur avait été agrandi au $\mathrm{XVI}^{\mathrm{e}}$ siècle sur les deux dernières travées de la nef, cette partie ainsi que l'escalier conduisant à l'autel furent démolie afin de rendre la crypte visible tandis qu'Annoni proposait une nouvelle solution pour relier la nef à la crypte et au chœur (fig. 8). L'abside fut alors complètement reprise, depuis les ouvertures jusqu'à la couverture en passant par 1'autel. Au cours de cette opération on découvrit des colonnes emmurées conservant les chapiteaux originaux ${ }^{45}$. Les chapiteaux mis en place par Zumaglini furent supprimés et remplacés par des moulures des restes découverts. On démolit les chapelles dont on considérait la présence nuisible à l'harmonie sévère de l'édifice, sans qu'elles présentent un intérêt esthétique et artistique particulier ${ }^{46}$. La conservation de certaines chapelles et l'abaissement du plafond du vaisseau central devait entrainer une solution problématique pour

\footnotetext{
${ }^{43}$ S. Muratori, « La chiesa dei funerali di Dante. S. Francesco in Ravenna », Rassegna d'Arte Antica e Moderna, 9, septembre 1921, p. 298 (traduction de l'auteur).

${ }^{44}$ G. Bovini, op. cit., 1964, p. 35.

${ }^{45}$ Ceux des colonnes de la nef avaient été détruits par Zumaglini et remplacé par des chapiteaux composites, M. G. Benini, op. cit., p. 40.

${ }^{46}$ C. Arpesani, «I restauri del S. Francesco di Ravenna », Arte Cristiana, 9, septembre 1921, p. 267-268.
} 
la couverture des collatéraux : les deux bas-côtés reçurent une toiture horizontale de hauteurs différentes ce qui détermina la forme asymétrique et peu heureuse de la façade ${ }^{47}$.

Pour renforcer l'atmosphère sévère, Annoni choisit de doter les fenêtres-hautes d'un verre opaque et de plonger la nef dans une semi pénombre, adoptant par là une solution que Ricci avait conçu pour conserver les fresques de San Vitale tout en diminuant leur visibilité. La découverte de fresques datant du début $\mathrm{du}_{\mathrm{XIV}}^{\mathrm{e}}$ siècle, notamment dans la chapelle des Da Polenta, répondit au désir des restaurateurs de retrouver l'image de l'église de Dante. L'émotion fut au comble lorsque, dans une paroi du vaisseau nord, apparut une figure à l'air pensif dans laquelle l'on voulut immédiatement reconnaître un portrait de Dante. Quand bien même Corrado Ricci et Santi Muratori se prononcèrent immédiatement contre cette interprétation, la découverte du « portrait de Dante » eut un retentissement dans la presse nationale, tandis que les habitants de Ravenne se pressaient en pèlerinage pour venir l'admirer ${ }^{48}$.

Grâce au soutien financier important du pape Benoît XV, le chantier fut achevé avant les célébrations de septembre 1921. Cette église avait bien peu de rapports avec l'édifice tel qu'il se présentait encore en 1917. Les travaux du centenaire dantesque conduisirent indéniablement à l'invention de l'église de Dante. Invention au double sens de création architecturale, produisant un état n'ayant jamais existé par le passé, mais aussi d'Inventio Sanctae Crucis, restauration vécue comme un chantier de fouille à la recherche de reliques du poète.

Le culte rendu à Dante devait s'étendre au-delà de son église à l'ensemble de la zone devenue un véritable sanctuaire du poète. Ludovico Pogliaghi et Ambrogio Annoni ornèrent le sépulcre construit par Camillo Morigia de matériaux précieux, tandis que l'artiste vénitien Umberto Bellotto concevait une grille de fer forgée pour l'enceinte dite de Braccioforte entourant cette tombe. A l'intérieur de cette enceinte, on érigea contre la façade du cloître un petit escalier au sommet duquel Annoni plaça une cloche offerte par l'ensemble des communes d'Italie. Sous cette cloche, fut aménagée l'entrée d'un petit «museo dantesco » composé d'un ensemble de vestiges des célébrations dédiées au poète qu'avait accueillies le sépulcre depuis la fin du XVIII ${ }^{\mathrm{e}}$ siècle. Bien davantage qu'un musée dédié à Dante, cette

\footnotetext{
${ }^{47}$ S. Muratori, op. cit., p. 298.

48 «la voce fu raccolta, riportata, diffusa, e in un baleno, tutti e tutti i ceti di persone incominciarono un pellegrinaggio che ancora continua [...] per venire a vedere Dante », ces paroles de Don Antonio Rotondi sont citées dans le journal Il Romagnolo du 17 janvier 1920.
} 
collection mettait en valeur le culte rendu au poète comme symbole de la nation et des luttes irrédentistes ${ }^{49}$. Euvre de propagande au sein duquel on pénétrait sous le signe de l'Italie unifiée (la cloche des communes), le musée de Dante devait perpétuer, au-delà des commémorations, le lien unissant le culte de Dante et les célébrations politiques de la ville de Ravenne, de l'Italie unifiée et de l'irrédentisme. Ce petit musée prenait place au premier étage de la galerie ouest du cloitre méridional qui fut également restauré. L'ensemble constitué par ce cloître, la tombe de Dante, l'enceinte de Braccioforte, le musée dantesque, l'église de Saint-François et la vaste place lui faisant face, composait ce qui fut rapidement appelé la zone dantesque de Ravenne (fig. 8).

Au-delà de Ravenne, Ricci avait réussi à transformer la première initiative de Mesini en un vaste programme de restauration sur l'ensemble du territoire italien ${ }^{50}$. Le fort sentiment nationaliste mis en jeu par le culte de Dante pouvait expliquer pourquoi, malgré une situation financière déplorable due aux dépenses de guerre et à la crise économique de 1920-1921, le gouvernement engagea des fonds considérables dans ces commémorations. L'Etat italien favorisa la dimension patriotique ${ }^{51}$ d'une fête qui devait être à la fois celle de la victoire et de l'unité nationale face aux profondes tensions de la société italienne et au sentiment largement diffusé dans la population d'une « victoire mutilée ${ }^{52} »$.

Si certains critères adoptés au cours des restaurations de 1921 apparaissent quelque peu en désaccord avec les conceptions théoriques formulées par Boito (ou parfois par Annoni luimême $)^{53}$, cette recherche du caractère médiéval des villes du centre et du nord de l'Italie devait s'intensifier durant la période mussolinienne ${ }^{54}$. A Ravenne les autorités fascistes reprirent le projet de la zone dantesque suivant la même veine néo-médiévale, aboutissant en

\footnotetext{
${ }^{49}$ Voir les références cités note 7 ainsi que B. Tobia, «La statuaria dantesca nell'Italia liberale : tradizione, identità e culto nazionale », dans Mélanges de l'Ecole française de Rome. Italie et Méditerranée, 109-1, 1997, p. 75-87.

${ }^{50}$ G. Mesini, op. cit., p. 10.

${ }^{51}$ ACS, AABBAA, divisione 1, 1908-1924, b. 1153, lettre du Directeur général des Antiquités et Beaux-arts au Ministre de l'Instruction Publique du 16 mai 1914. Une loi spéciale fut adoptée par le Parlement soulignant le « spiccato carattere di solennità nazionale » des commémorations. Loi nº71, Celebrazione del VI centenario della morte di Dante, 17 novembre 1920.

${ }_{52}$ L'expression est de D'Annunzio et souligne le sentiment d'avoir été lésé par le Traité de Versailles, notamment dans les revendications territoriales de l'Italie.

${ }^{53}$ Toutefois notre but n'étant pas d'émettre un jugement sur la valeur de ces restaurations, nous nous sommes bien gardés de les replacer dans les catégories du schéma progressiste généralement employé par les historiens de la restauration en Italie. Pour une critique de cette historiographie, voir A. M. Racheli, Restauro e architettura, Teoria e critica del restauro architettonico e urbano dal XVIII al XXI secolo, Rome, Gangemi, 2006, p. 76-77.

${ }^{54}$ Pour une étude de stratégies similaires en Toscane durant la période fasciste, voir M. D. Lasansky, The Renaissance perfected: architecture, spectacle and tourism in fascist Italy, University Park, Pennsylvania state university, 2004.
} 
1936 à l'inauguration en grande pompe par Mussolini de ce qui était entre-temps devenu la zona del silenzio ${ }^{55}$. Cette continuité semble confirmer à posteriori que le choix de commémorer le poète par la mise en valeur d'une certaine architecture, restaurée parfois jusqu'à la réinvention, ne peut se comprendre que dans le cadre des enjeux patriotiques et nationalistes du patrimoine.

Thomas Renard prépare une thèse sur L'architecture de l'Unité d'Italie (1861-1922), entre recherche d'un style national et affirmation des identités régionales aux universités de Paris IV et IUAV de Venise, sous la codirection de M. Claude Mignot et de M. Guido Zucconi

\footnotetext{
${ }^{55}$ Voir P. Bolzani (dir.), Arata e Ravenna, Opere e progetti nella città di Corrado Ricci, Ravenne, Longo, 2008.
} 